\title{
Characteristics of DSSC Panels with Silicone Encapsulant
}

\author{
Jun-Gu Kang, ${ }^{1}$ Jin-Hee Kim, ${ }^{2}$ Han-Bin Jang, ${ }^{3}$ and Jun-Tae Kim ${ }^{4}$ \\ ${ }^{1}$ Graduate School, Department of Architectural Engineering, Kongju National University, 275 Budae-dong, \\ Cheonan, Chungnam 330-717, Republic of Korea \\ ${ }^{2}$ Green Home Energy Technology Research Center, Kongju National University, 275 Budae-dong, Cheonan, \\ Chungnam 330-717, Republic of Korea \\ ${ }^{3}$ Graduate School, Department of Energy System Engineering, Kongju National University, 275 Budae-dong, \\ Cheonan, Chungnam 330-717, Republic of Korea \\ ${ }^{4}$ Department of Architectural Engineering, Kongju National University, 275 Budae-dong, \\ Cheonan, Chungnam 330-717, Republic of Korea \\ Correspondence should be addressed to Jun-Tae Kim; jtkim@kongju.ac.kr
}

Received 18 July 2014; Accepted 19 January 2015

Academic Editor: Mohammad Muneer

Copyright (C) 2015 Jun-Gu Kang et al. This is an open access article distributed under the Creative Commons Attribution License, which permits unrestricted use, distribution, and reproduction in any medium, provided the original work is properly cited.

\begin{abstract}
Dye-sensitized solar cells (DSSC) allow light transmission and the application of various colors that make them especially suitable for building-integrated PV (BIPV) application. In order to apply DSSC modules to windows, the module has to be panelized: a DSSC module should be protected with toughened glass on the entire surface. Up to the present, it seems to be common to use double glazing with DSSC modules, with air gaps between the glass pane and the DSSC modules. Few studies have been conducted on the characteristics of various glazing methods with DSSC modules. This paper proposes a paneling method that uses silicone encapsulant, analyzing the performance through experimentation. Compared to a multilayered DSSC panel with an air gap, the encapsulant-applied panel showed 6\% higher light transmittance and 7\% higher electrical efficiency. The encapsulant also prevented electrolyte leakage by strengthening the seals in the DSSC module.
\end{abstract}

\section{Introduction}

1.1. Background. Dye-sensitized solar cells (DSSC) are known to allow light transmission and can be manufactured in various colors and shapes. They are also relatively less sensitive to angle of incidence. These characteristics of the cells make them especially suitable for building-integrated photovoltaic (BIPV). Large-size PV modules seem to be more practical for building applications. However, the technological limitations of DSSCs restrict their use to smaller sizes of modules. Currently, the available size of DSSC modules is $300 \times$ $300 \mathrm{~mm}$ at commercial scale, even though there have been efforts to make larger DSSC modules. These issues make it problematic to apply DSSC modules to buildings. For a DSSC module to be a replacement for existing envelope elements, it should be panelized with several modules to make a building component unit that can comply with industry practices. There are two different methods of panelization with DSSC modules: connected individual modules that are supported in a large-size frame and panelized modules with external panes of toughened glass. However, the lattice structure with the former method seems not to be suitable for DSSC panels in terms of wiring, energy efficiency, safety, and visibility. The soda lime glass typically used for the modules is too weak to use as a finishing material; if the glass is damaged, glass shards may also pose a danger.

Due to these issues, DSSC modules are typically panelized in double glazing unit types with external panes of toughened glass.

Panelization is the placement of a DSSC module with air gaps between the glass panes, creating a triple glazing unit (TGU) panel, as can be seen in Figure 1. In this configuration, more solar radiation is reflected from the panel due to the difference in refractive indices between the air and the glass, so that the amount of solar radiation transmitted to the DSSC module can be reduced. This structural characteristic of the panel decreases the solar energy gain, which in turn reduces the electrical efficiency of the PV modules. 


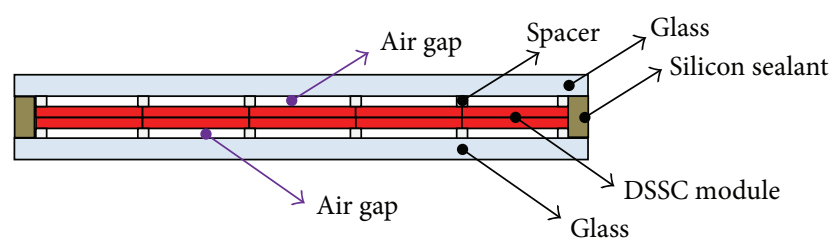

FIGURE 1: Triple glazing unit type DSSC panel.

This type of panel also incorporates a provision for an edge sealant similar to that used in multilayered glazing units. It is hard to protect the inside of the DSSC module from external stress. The electrolyte is a key component of the DSSC. An electrolyte containing a redox couple fills the gap between the electrodes. Usually, an iodide/triiodide redox couple dissolved in organic solvents is used for the electrolyte. Although iodide/triiodide has been demonstrated as the redox couple for regeneration of the oxidized dye, its severe corrosion for many sealing materials, especially metals, causes a difficult assembling and sealing for a largearea DSSC and poor long-term stability of DSSC [1]. There is also a concern that corrosion of the connected electrodes between the modules could cause efficiency reduction of the $\operatorname{DSSC}[2,3]$.

1.2. Objective. To solve the issues involved with the current method of DSSC paneling with double layers of glazing, this study proposes a method that uses silicone encapsulants between the DSSC module layer and the glass panes. The electrical performance and properties of this encapsulant type of DSSC panel were analyzed and were compared with those of the triple glazing type panel.

\section{Literature and Theoretical Review}

2.1. DSSC Panel. With regard to research on the scale-up of DSSCs, Dai et al. [4] reported panel development with a size of $450 \mathrm{~mm} \times 800 \mathrm{~mm}$; this panel consisted of 12 DSSC modules with sizes of $150 \mathrm{~mm} \times 200 \mathrm{~mm}$. The researchers utilized commercially available module units for panelization and reported an experimental performance with the same capacity as that of a $500 \mathrm{Wp}$ DSSC PV system. In 2004, Tulloch [5] proposed a method for DSSC modules and panel designs applicable to buildings. To upscale DSC sizes, three different module designs have been considered: the parallel grids module, series interconnect $\mathrm{Z}$, and $\mathrm{W}$ designs module, monolithic series interconnect. And using a $600 \mathrm{~mm} \times$ $900 \mathrm{~mm}$ panel as a standard size and suggesting a panelization method to produce panels in the form of multilayered glazing units, the number of modules required to fit the $600 \mathrm{~mm} \times$ $900 \mathrm{~mm}$ panel is 24, in three rows of eight modules.

Hinsch et al. [6] reported on the results of the German project ColorSol. The project focuses on the application field of BIPV. Semitransparent DSC modules (ColorSol) with internal interconnection have been developed using glass frit-sealing technology. Proto types of glass façade elements $(70 \mathrm{~cm} \times 200 \mathrm{~cm})$ have been developed which consist of several serially interconnected DSC modules each with a size of $30 \mathrm{~cm} \times 30 \mathrm{~cm}$. Outdoor measurements on such modules have been performed, which confirmed a module efficiency of $4.2 \%$ on the active module area under full sunlight conditions.

Kang et al. developed a prototype of transparent DSSC windows and reported the results of their performance. Their DSSC cells had a transmittance of approximately $60 \%$ in the visible range; the open-circuit voltage, $V_{\mathrm{oc}}$, and the short-circuit photocurrent, $J_{\mathrm{sc}}$, of the cells were $0.64 \mathrm{~V}$ and $250 \mathrm{~mA} / \mathrm{cm}^{2}$, respectively. It was found that the transparent $\mathrm{PV}$ window composed of nine DSSC modules in series generating a $V_{\mathrm{oc}}$ of $5.7 \mathrm{~V}$ and a $J_{\mathrm{sc}}$ of $220 \mathrm{~mA} / \mathrm{cm}^{2}$ under 1 sun condition [7].

It is a great challenge for DSSC modules and their panels to maintain durability. The durability of large-size DSSC panels seems to depend on the sealing of each DSSC module. Therefore, recent research on module and panel durability has been reported. Sastrawan et al. [8] proposed glass frit as sealing material for DSSC modules. Glass frit is thermally, mechanically, and chemically very stable and can be applied via screen printing. DSSC modules with dimensions of $300 \mathrm{~mm} \times 300 \mathrm{~mm}$ with glass frit as primary sealing material have been produced. It has been shown that glass frit is applicable for the upscaling of DSSC technology to large area panels. The thermal stability of the glass frit sealing and the integrated series connections were verified in a thermal cycling experiment. In 2012, Ribeiro et al. [9], through the development of a laser-assisted glass frit sealing process, presented a sealing method for improving DSSC durability beyond what had been possible using the previous method. On the other hand, the introduction of volatile solvents to the electrolyte in combination with imperfect sealing frequently resulted in leakage and evaporation of the electrolyte at elevated temperatures. Solvents used as electrolytes for DSSC modules in turn decrease the life-span and efficiency of electrodes. In order to solve these problems, instead of using volatile solvents, studies have been conducted on various solid electrolytes or quasisolid electrolytes. Yoon et al. [10] analyzed the long-term stability of DSSCs using ion gel electrolytes; they reported that the photocurrent and conversion efficiency obtained using such materials were higher than those possible when using ionic liquids.

2.2. Optical Characteristics of DSSC Panel. The electrical generation of PV systems depends on the amount of incoming solar radiation on PV modules; the variation in the amount of radiation that hits the PV surface can be described using optical principles, such as refraction, reflection, and absorption. Refraction, a phenomenon that changes the propagation direction of light when light passes through media with different wavelengths, may cause reflection to occur depending on the incidence angle and refractive index of the mediums.

In order to optimize the efficiency of solar cells and PV modules, it is important for PV module designers to understand these optical losses. In 2012, Khoo et al. [11] suggested a method to quantify the optical loss related to the cover glass and encapsulant of PV modules. In order to optimize the performance of PV modules, it is necessary to 


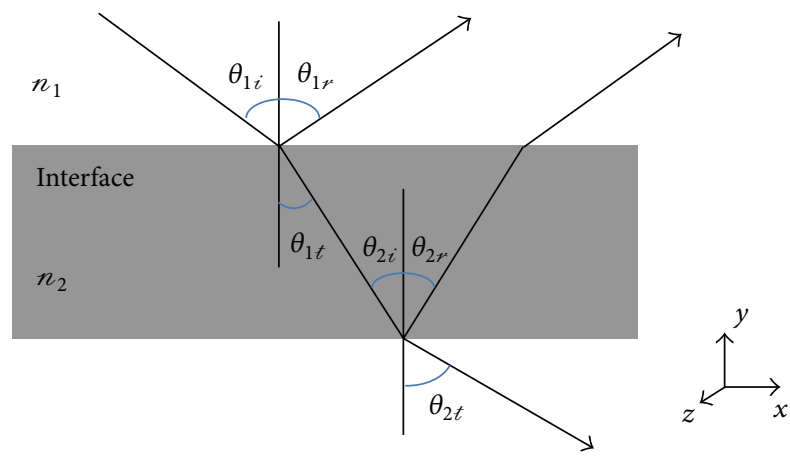

FIGURE 2: Reflection and transmission of light at an interface.

maximize the light transmittance. According to the study by Powell et al. [12], the electrical efficiency of PV modules with silicon encapsulant was improved by $0.31-1.45 \%$ compared to that of modules with conventional ethylene vinyl acetate (EVA). This result indicates that the use of optically superior silicone encapsulant materials is helpful for PV module energy performance.

Moreover, Norris et al. [13] reported that by changing the silicone encapsulant refractive index from 1.38 to 1.54 using the chemical composition, the PV efficiency was improved by 0.5 to $2.5 \%$ when applying silicone encapsulant materials instead of EVA.

As can be seen in Figure 2, it is typical that when sunlight passes through a medium, reflection occurs at the border; a portion of the light is refracted into the medium; this light, at the second medium border, is reflected once again before emerging on the other side. Light that is refracted passes through the medium. In Figure $2, n_{1}, n_{2}$ are the refractive indices of the two mediums and $\theta_{i}, \theta_{t}$, and $\theta_{r}$ are the incidence angle, refractive angle, and reflection angle, respectively.

The dynamic relationship between reflected and refracted light is extremely complex but can be simplified using Fresnel's equation and Snell's law [14].

Snell's law can be expressed as in (1) using the incidence and refractive angles of two different mediums. Cosnider

$$
n_{1} \sin \theta_{i}=n_{2} \sin \theta_{t}
$$

Fresnel's equation separately expresses the reflection coefficient and transmission coefficient of light entering a medium border [15]. Either reflection or transmission occurs with parallel or perpendicular polarization.

The incidence and refraction angles of different mediums have trigonometric relationships, and the reflection coefficients $\left(r_{\|}, r_{\perp}\right)$ and transmission coefficients $\left(t_{\|}, t_{\perp}\right)$ of parallel and perpendicular polarized light are expressed in

$$
\begin{aligned}
& r_{\|}=\frac{n_{2} \cos \theta_{i}-n_{1} \cos \theta_{t}}{n_{1} \cos \theta_{t}-n_{2} \cos \theta_{i}}, \\
& r_{\perp}=\frac{n_{1} \cos \theta_{i}-n_{2} \cos \theta_{t}}{n_{1} \cos \theta_{i}+n_{2} \cos \theta_{t}},
\end{aligned}
$$

$$
\begin{aligned}
& t_{\|}=\frac{2 n_{1} \cos \theta_{i}}{n_{1} \cos \theta_{t}+n_{2} \cos \theta_{i}}, \\
& t_{\perp}=\frac{2 n_{1} \cos \theta_{i}}{n_{1} \cos \theta_{i}+n_{2} \cos \theta_{t}} .
\end{aligned}
$$

When light hits normal to the medium border $\left(\cos \theta_{i}=\right.$ $\left.\cos \theta_{t}=1\right)$, Fresnel's equation and Snell's law can be simplified into (3), which are the reflection coefficient $(r)$ and transmission coefficient $(t)$ in terms of refractive indices. Consider

$$
\begin{aligned}
& r=\frac{n_{2}-n_{1}}{n_{2}+n_{1}}, \\
& t=\frac{n_{2}-n_{1}}{n_{2}+n_{1}} .
\end{aligned}
$$

The reflection rate $(R)$ can be defined using the reflection coefficient $(r)$. The reflection rate is a ratio of reflected light to the strength of incoming light, which is the square of the reflection coefficient. For light incoming at a normal angle, the equation for the reflection rate is given by

$$
R=\mu^{2}=\left(\frac{n_{2}-n_{1}}{n_{2}+n_{1}}\right)^{2} .
$$

As a result, the more similar the refraction indices are, the more the reflection rate will decrease.

2.3. DSSC Encapsulation. The encapsulant used in the PV module is adhesively attached to each layer, protecting the solar cells from external shock. It is advantageous to use a transparent encapsulant that allows high light transmission.

The encapsulant used in a DSSC panel should have a low setting temperature and a high transmittance similar to that of EVA; it should be made of materials capable of protecting the cells from external humidity or shock. Such materials are acryl, epoxy, and silicon-type materials, of which siliconbased materials do not carry the risk of discoloration due to ultraviolet radiation. Hence, this paper applied a siliconbased encapsulant in the DSSC panelization process.

\section{Experiment}

3.1. DSSC Panel Design. For this study, two different test models of DSSC panel were made, as shown in Figure 3: (a) TGU type and (b) silicon encapsulant type. We analyzed the electrical performance and durability of these DSSC panels. Table 1 specifies each layer of both panels. Each DSSC module has a size of $0.9 \mathrm{~m}^{2}$ and consists of 23 DSSCs connected in series. For the DSSC, N719 dye, soda lime glass covered with $5 \mu \mathrm{m}$ transparent conductive film $\left(\mathrm{TiO}_{2}\right)$, and a 3methoxypropionitrile (MPN) electrolyte were used.

Silicon encapsulant type is made by inserting silicon with syringe into air gap between DSSC module and exterior glass and finishing the edge of exterior glass with silicon sealant in TGU panel structure (Figure 1). A two-component silicone liquid that is commonly used was applied as the 

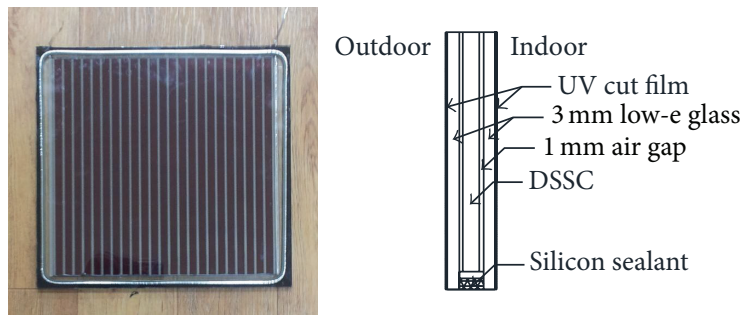

(a) Triple glazing unit type
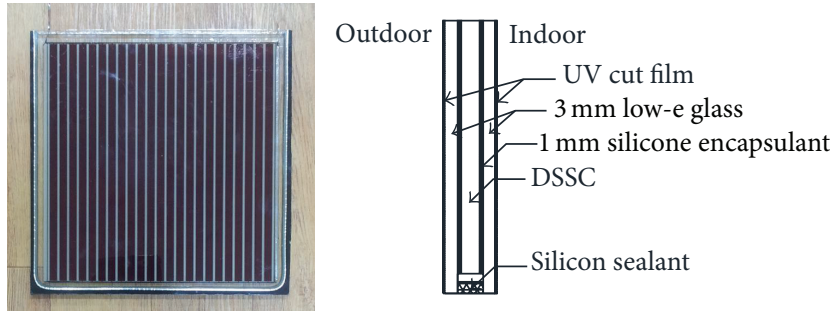

(b) Silicone encapsulant type

FIGURE 3: DSSC panel configurations.

TABLE 1: DSSC panel structure.

\begin{tabular}{lcc}
\hline Panel type & Thickness $(\mathrm{mm})$ & Layers \\
\hline Triple glazing unit type & 12.8 & UV cut film $+3 \mathrm{~mm} \mathrm{CL}+1 \mathrm{~mm}$ Air $+4.4 \mathrm{~mm} \mathrm{DSSC}+1 \mathrm{~mm} \mathrm{Air}+3.2 \mathrm{~mm}$ CL \\
Silicone encapsulant type & 12.8 & UV cut film $+3 \mathrm{~mm} \mathrm{CL}+1 \mathrm{~mm}$ silicone $+4.4 \mathrm{~mm} \mathrm{DSSC}+1 \mathrm{~mm}$ silicone $+3.2 \mathrm{~mm}$ CL \\
\hline
\end{tabular}

${ }^{*}$ CL: clear glass. ${ }^{*}$ Air: air space.

TABLE 2: Characteristics of silicone filler.

\begin{tabular}{lccc}
\hline Type & $\begin{array}{c}\text { Refractive } \\
\text { index }\end{array}$ & $\begin{array}{c}\text { Viscosity } \\
(\mathrm{cp})\end{array}$ & $\begin{array}{c}\text { Setting time } \\
\text { (hour) }\end{array}$ \\
\hline $\begin{array}{l}\text { Two-component } \\
\text { silicone liquid }\end{array}$ & 1.43 & 1,000 & $8\left(25^{\circ} \mathrm{C}\right)$ \\
\hline
\end{tabular}

encapsulant. Being a type that sets at room temperature, it had the advantage of being able to protect the DSSC from thermal shock; it also had a low viscosity, making it easy to apply. Additionally, to minimize the reflection of solar radiation, a silicone encapsulant with a refractive index of 1.43, similar to that of glass (1.5), was used. Table 2 summarizes the properties of the encapsulant used.

3.2. Testing Methods. The transmission, electrical, and electrolyte-proofing performances were analyzed. Light transmission at different spectrums was measured using a Shimadzu UV-3600 spectrophotometer (Figure 4).

To measure the electrical performance, an unpaneled DSSC module, a TGU type DSSC panel, and a silicone encapsulant type DSSC panel were all tested. A solar simulator, as can be seen in Figure 5, was used to create a standard light source at 1.5 air mass (AM) ( $1 \mathrm{sun}, 100 \mathrm{~mW} / \mathrm{cm}^{2}$ ), after which the $I-V$ curve was measured. An outdoor test was also performed to measure the generation performance of each panel type.

To determine the device's ability to prevent electrolyte leakage under high temperature, a thermal stress analysis

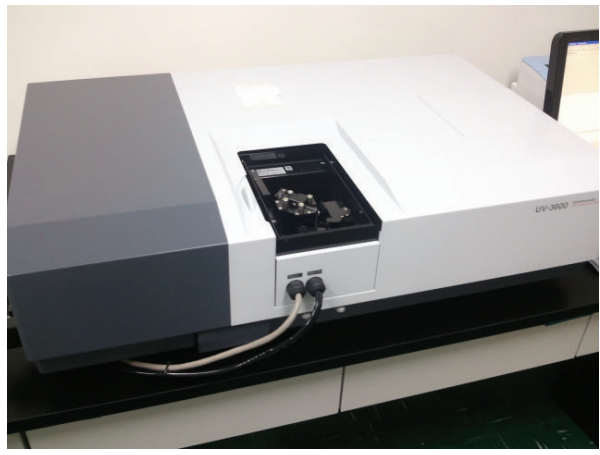

Figure 4: UV-Vis spectrophotometer.

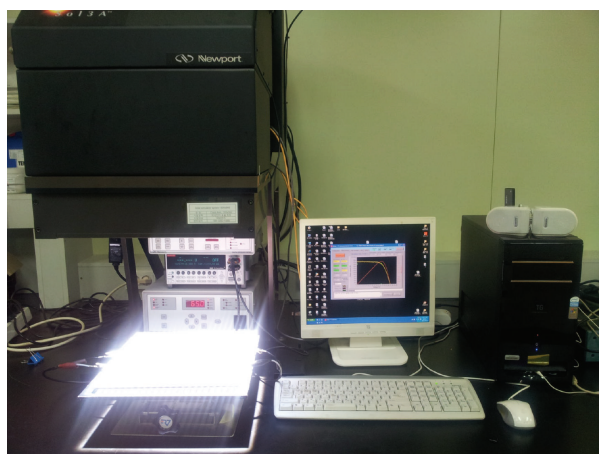

FIGURE 5: Solar simulator used for DSSC module and panels. 


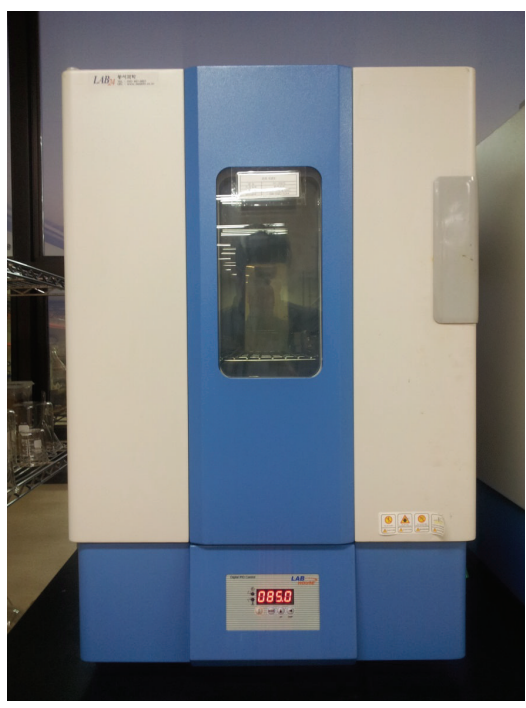

FIGURE 6: Heat test chamber.

was conducted; the adhesiveness of the encapsulant was also tested. For this testing purpose, a heat test chamber, shown in Figure 6, was used to measure the leaked fluid of each panel type under the conditions of $85 \pm 2^{\circ} \mathrm{C}$ temperature for 1000 hours.

To measure the amount of electrolyte leakage, we photographed the surface of module of DSSC every 24 hours, scanned the image, and finally measured the area by CAD. We assumed that the area of module filled with electrolyte is $100 \%$ and calculated the amount of leakage by the area changed according to the leakage of electrolyte.

\section{Results and Analysis}

4.1. Light Transmission Performance. The optical performance of the DSSC panel was analyzed using transmittance measurements. Transmittance values by panel type showed similar patterns to those shown in Figure 7, and light did not pass through in the short UV wavelength range up to $380 \mathrm{~nm}$. Transmittance in the visible light spectrum $(380 \mathrm{~nm} \sim 780 \mathrm{~nm})$, which directly affects the electrical performance of DSSC modules, was found to decrease after rising within the range of $680 \mathrm{~nm} \sim 730 \mathrm{~nm}$. Panels with silicone encapsulant materials had a maximum transmittance of $37 \%$, while the TGU panels had a maximum transmittance of $31 \%$. In the visible light wavelength, panels with the encapsulant had an average transmittance of $19.2 \%$, which was $3.1 \%$ higher than that of the TGU panels.

This discrepancy can be explained using the calculation of reflectivity (4) in Fresnel's equation. The reflectivity for TGU panels was found to be $4 \%$ when calculated with refractive indices of 1.5 for glass and 1.0 for air. On the other hand, the reflectivity of the panels with the encapsulant was $0.06 \%$ when calculated with 1.5 as the refractive index for glass and 1.43 as the refractive index for the encapsulant. This indicates that the introduction of the silicone encapsulant leads to a $3.94 \%$ decrease in reflectivity of the panels compared to that of the conventional TGU panel.

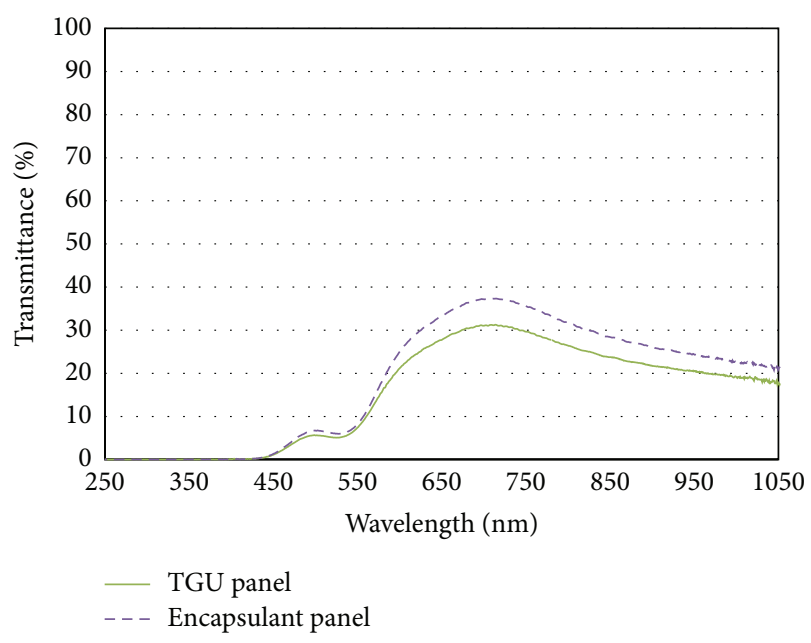

FIGURE 7: Transmittance of the TGU DSSC panel and the silicone encapsulant DSSC panel.

TABLE 3: Electrical efficiency per panel type and DSSC module.

\begin{tabular}{lcccc}
\hline Type & $\begin{array}{c}I_{\text {sc }} \\
(\mathrm{mA})\end{array}$ & $\begin{array}{c}V_{\text {oc }} \\
(\mathrm{V})\end{array}$ & $\begin{array}{c}\text { FF } \\
(\%)\end{array}$ & $\begin{array}{c}\text { Eff } \\
(\%)\end{array}$ \\
\hline DSSC module & 304.3 & 16.52 & 62.7 & 5.13 \\
TGU panel & 265.5 & 16.34 & 64.0 & 4.52 \\
Encapsulant panel & 286.4 & 16.52 & 63.2 & 4.88 \\
\hline
\end{tabular}

While TGU type panels had higher reflectivity due to the difference in refractive index between glass and air, panels with encapsulant obtained lower reflectivity by introducing a material that had a refractive index similar to that of glass, thus improving the optical performance of the DSSC panels.

4.2. Electrical Performance. The electrical efficiency of each panel type, listed in Table 3, showed that the unpaneled DSSC modules yielded 5.13\% efficiency, the TGU type $4.52 \%$, and the encapsulant type $4.88 \%$. The reduction in efficiency as a result of paneling was confirmed with these test results. Compared to the unpaneled module, the TGU DSSC panels experienced an $11.89 \%$ efficiency decrease, while the encapsulant type panel saw a reduced efficiency of only $4.87 \%$. The DSSC panel encapsulated with silicone showed an improvement in electrical efficiency. Considering the $I$ $V$ curves shown in Figure 8, compared to the TGU panel, the encapsulant type DSSC panel had increased the current values. Through these results, it was shown that increasing the light transmittance of the panel using clear silicone encapsulant also increased the DSSC electrical efficiency.

Outdoor tests were conducted on September 24th, 2012. The DSSC module and DSSC panels were installed as shown in Figure 9, facing south at a $45^{\circ}$ angle at the location of $36.88^{\circ} \mathrm{N}$ latitude and $127.18^{\circ} \mathrm{E}$ longitude. In Figure 10 , the amount of power produced by the TGU panel can be seen to be $15.79 \mathrm{Wh}$, while the encapsulant panel produced $17.54 \mathrm{Wh}$ of power; this shows a $10 \%$ improvement in performance due to the encapsulant use for the panel. 


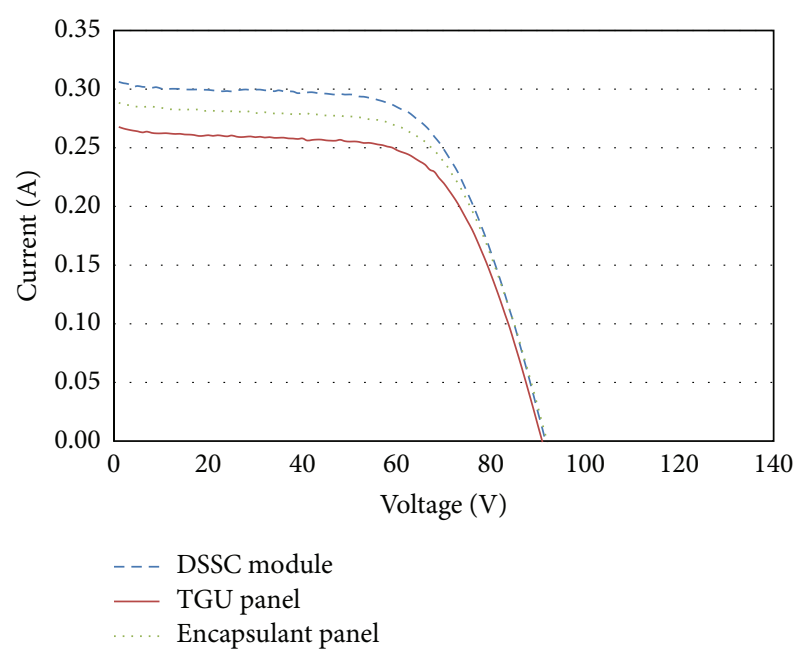

FIGURE 8: $I$ - $V$ curves of the DSSC module and panels.

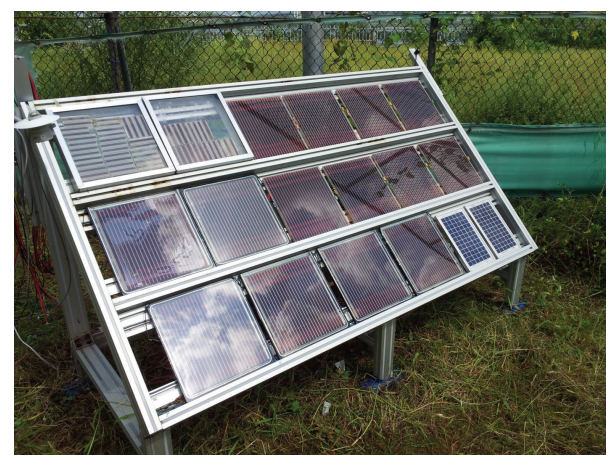

FIGURE 9: Outdoor testing facility.

4.3. Electrolyte Leakage Prevention Performance. Testing the TGU panel, electrolyte leakage was found to occur at a rate of $100 \%$ within 80 hours of use, which is noted in Figure 11. The encapsulant panels, on the other hand, recorded only a $62 \%$ leakage rate in 1000 hours of operation. These testing results show that the encapsulant panel is superior to the TGU type panels in preventing electrolyte leakage. It is believed that the silicone encapsulant between the glass panes and the DSSC modules works as a supportive sealing agent around the modules. Hence, it is expected that applying an encapsulant will increase the durability of the panel.

\section{Conclusion}

This paper has proposed a DSSC panelization method using silicone encapsulants; we analyzed the performance of the proposed panels compared to that of existing TGU type panels in terms of light transmittance and electrical and electrolyte leakage prevention performance.

It was found that the proposed encapsulant type performed with $6 \%$ higher transmittance, $7 \%$ higher electrical efficiency, and $10 \%$ higher electric generation than those

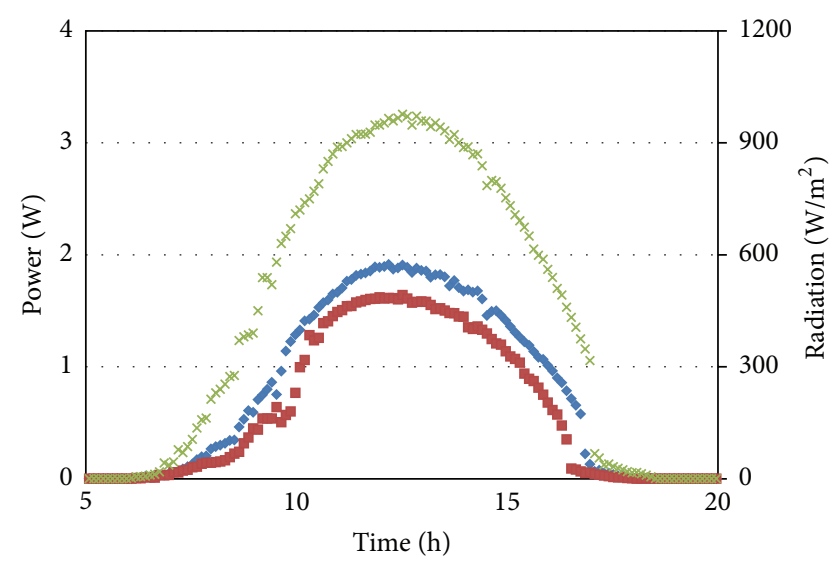

- Encapsulant panel
- TGU panel
$\times$ Radiation

FigURE 10: Electricity generation of the panels.

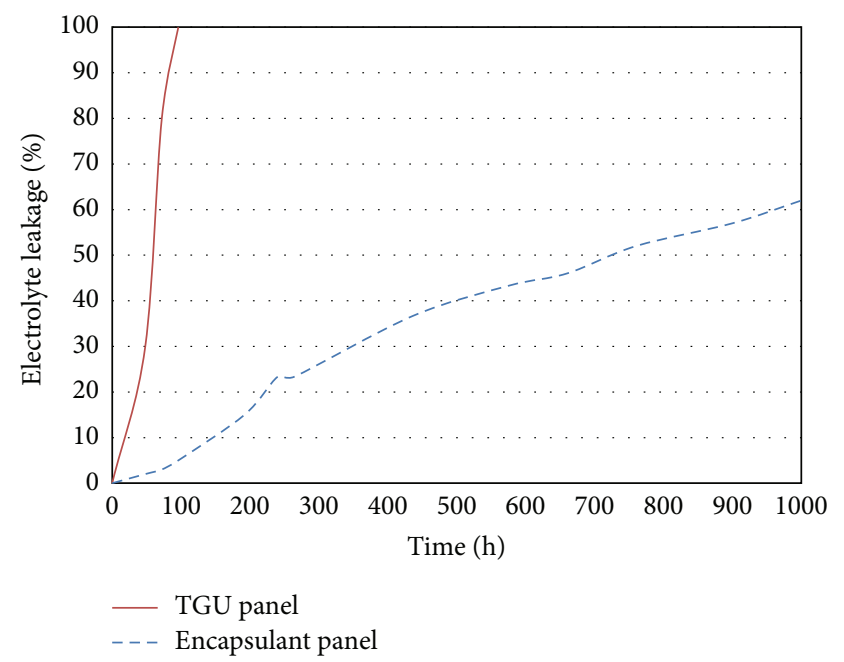

FIGURE 11: Electrolyte leak rate over time for the panels.

values for conventional TGU panels. Additionally, the encapsulant added to the panel strengthens the seals of the DSSC modules, preventing electrolyte leakage.

It was also determined that paneling should be used with an encapsulant that has a refractive index similar to that of the panel material, as well as one that has a high transmittance.

Further work to validate the properties of encapsulants in varied environments may be required to establish optimized encapsulated DSSC panel designs. The encapsulants' influence on the insulation values of the DSSC panels as building envelope components and the electrical performance of scale-up DSSC panels according to series interconnections of the DSSC modules will be included in further studies.

\section{Conflict of Interests}

The authors declare no conflict of interests. 


\section{Acknowledgments}

This work was supported by the Energy Efficiency \& Resources of the Korea Institute of Energy Technology Evaluation and Planning (no. 2012T100100065) and by the Human Resources Development Program (no. 20134010200540) of the Korea Institute of Energy Technology Evaluation and Planning (KETEP) grant funded by the Korea Government Ministry of Trade, Industry and Energy.

\section{References}

[1] J. Wu, Z. Lan, S. Hao et al., "Progress on the electrolytes for dyesensitized solar cells," Pure and Applied Chemistry, vol. 80, no. 11, pp. 2241-2258, 2008.

[2] Z. Yu, N. Vlachopoulos, M. Gorlov, and L. Kloo, "Liquid electrolytes for dye-sensitized solar cells," Dalton Transactions, vol. 40, no. 40, pp. 10289-10303, 2011.

[3] G. H. Kang, J. H. Park, G. J. Yu, H. G. An, and D. Y. Han, "The effect of electrical properties with degradation of university of science and technology EVA sheet and electrode in photovoltaic module," in Proceedings of the Summer Conference of the Korean Institute of Electrical and Electronical Material Engineers, pp. 2628, 2005.

[4] S. Dai, J. Weng, Y. Sui et al., "The design and outdoor application of dye-sensitized solar cells," Inorganica Chimica Acta, vol. 361, no. 3, pp. 786-791, 2008.

[5] G. E. Tulloch, "Light and energy-dye solar cells for the 21st century," Journal of Photochemistry and Photobiology A: Chemistry, vol. 164, no. 1-3, pp. 209-219, 2004.

[6] A. Hinsch, H. Brandt, W. Veurman et al., "Dye solar modules for facade applications: recent results from project ColorSol," Solar Energy Materials \& Solar Cells, vol. 93, no. 6-7, pp. 820824, 2009.

[7] M. G. Kang, N.-G. Park, Y. J. Park, K. S. Ryu, and S. H. Chang, "Manufacturing method for transparent electric windows using dye-sensitized $\mathrm{TiO}_{2}$ solar cells," Solar Energy Materials and Solar Cells, vol. 75, no. 3-4, pp. 475-479, 2003.

[8] R. Sastrawan, J. Beier, U. Belledin et al., "A glass frit-sealed dye solar cell module with integrated series connections," Solar Energy Materials \& Solar Cells, vol. 93, pp. 820-824, 2009.

[9] F. Ribeiro, J. MaçAira, R. Cruz, J. Gabriel, L. Andrade, and A. Mendes, "Laser assisted glass frit sealing of dye-sensitized solar cells," Solar Energy Materials \& Solar Cells, vol. 96, no. 1, pp. 4349, 2012.

[10] J. S. Yoon, D. K. Kang, J. O. Won, J.-Y. Park, and Y. S. Kang, "Dye-sensitized solar cells using ion-gel electrolytes for longterm stability," Journal of Power Sources, vol. 201, pp. 395-401, 2012.

[11] Y. S. Khoo, T. M. Walsh, and A. Gaberle, "Novel method for quantifying optical losses of glass and encapsulant materials of silicon wafer based PV modules," Energy Procedia, vol. 15, pp. 403-412, 2012.

[12] N. E. Powell, B. K. Hwang, A. W. Norris, B. M. Ketola, G. Beaucarne, and K. R. McIntosh, "Improved spectral response of silicone encapsulanted photovoltaic modules," in Proceedings of the 35th IEEE Photovoltaic Specialists Conference (PVSC '10), pp. 2791-2794, Honolulu, Hawaii, USA, June 2010.

[13] A. Norris, N. Powell, B. Ketola, J. N. Cotselland, and K. R. McIntosh, "Advanced silicone photovoltaic encapsulants," Future Photovoltaics, pp. 1-8, 2010.
[14] Wikipedia article, "Snell's law," http://en.wikipedia.org/wiki/ Snell_law.

[15] Wikipedia Article, "Fresnel equations," June 2014, http://en .wikipedia.org/wiki/Fresnel_equations. 

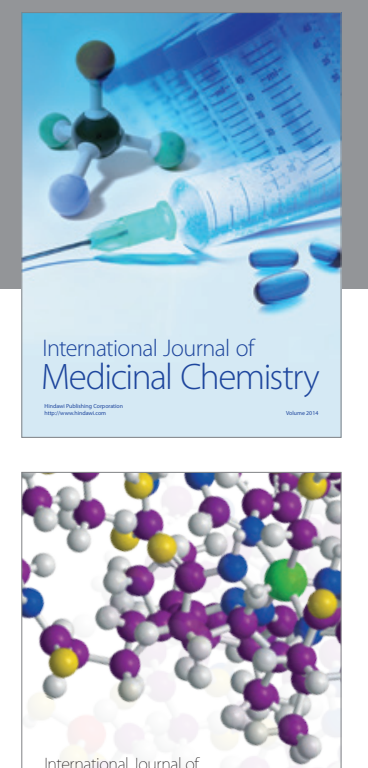

\section{Carbohydrate} Chemistry

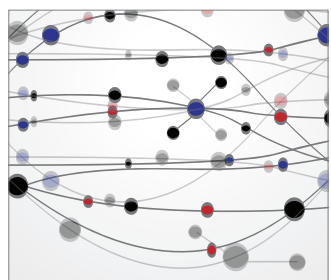

The Scientific World Journal
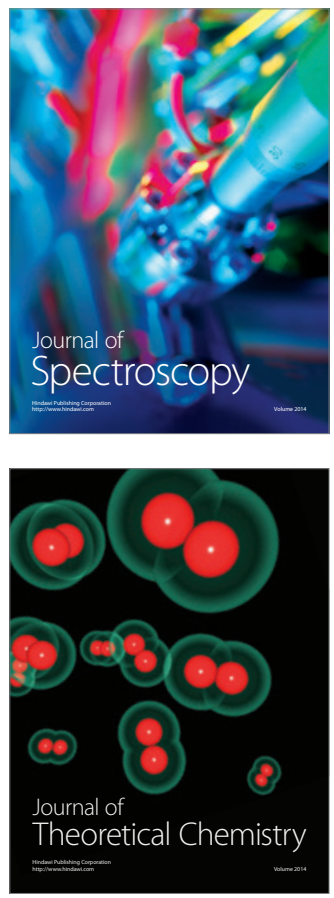
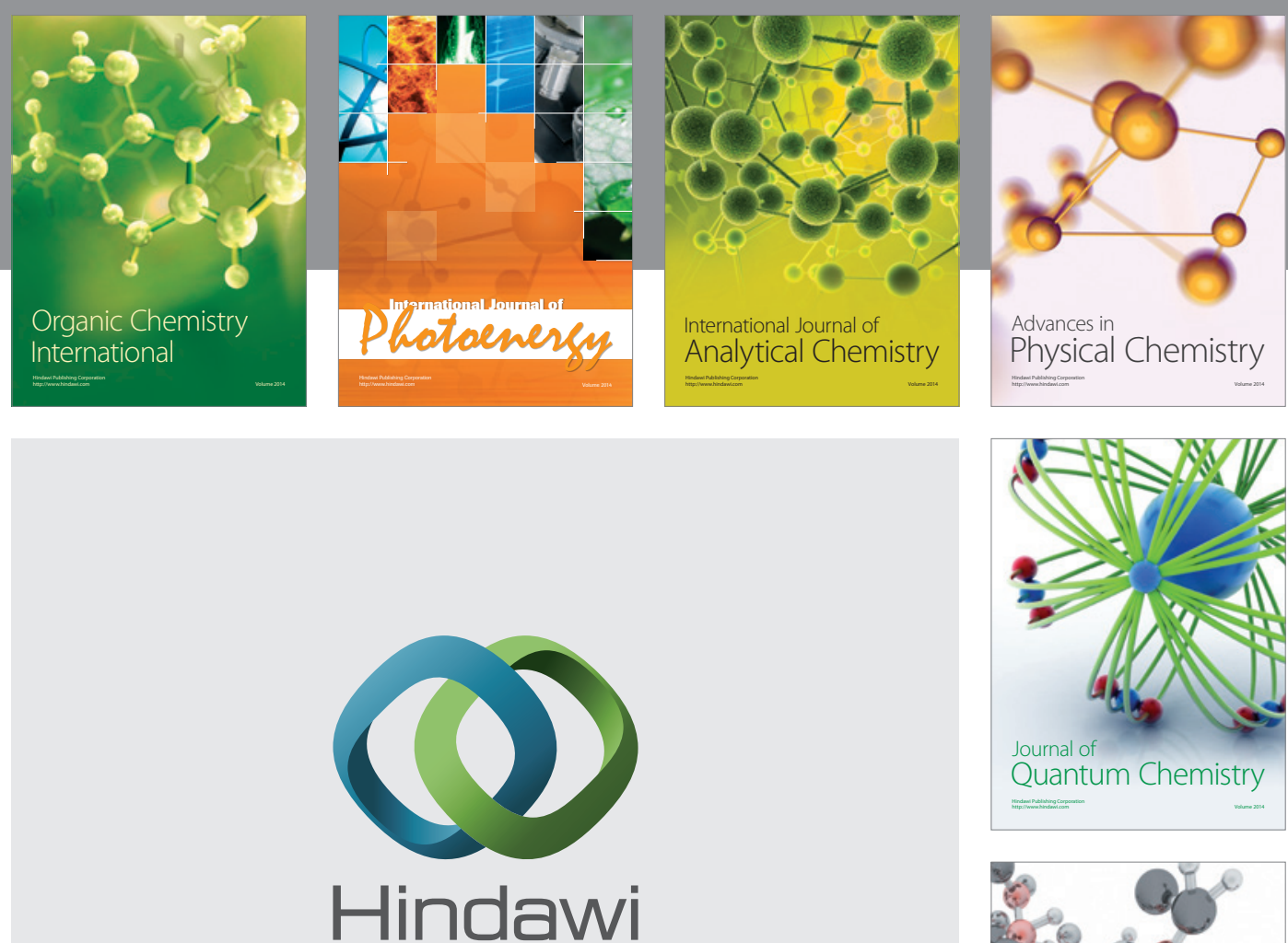

Submit your manuscripts at

http://www.hindawi.com

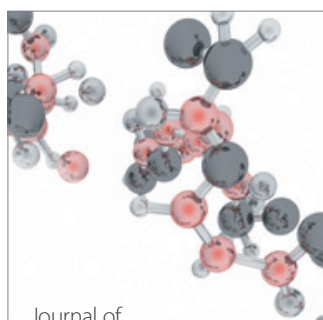

Analytical Methods

in Chemistry

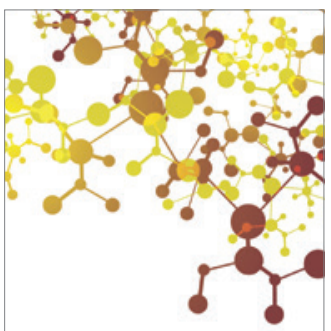

Journal of

Applied Chemistry

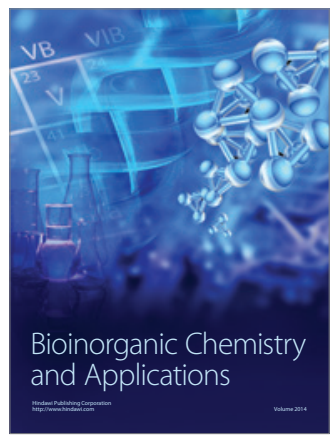

Inorganic Chemistry
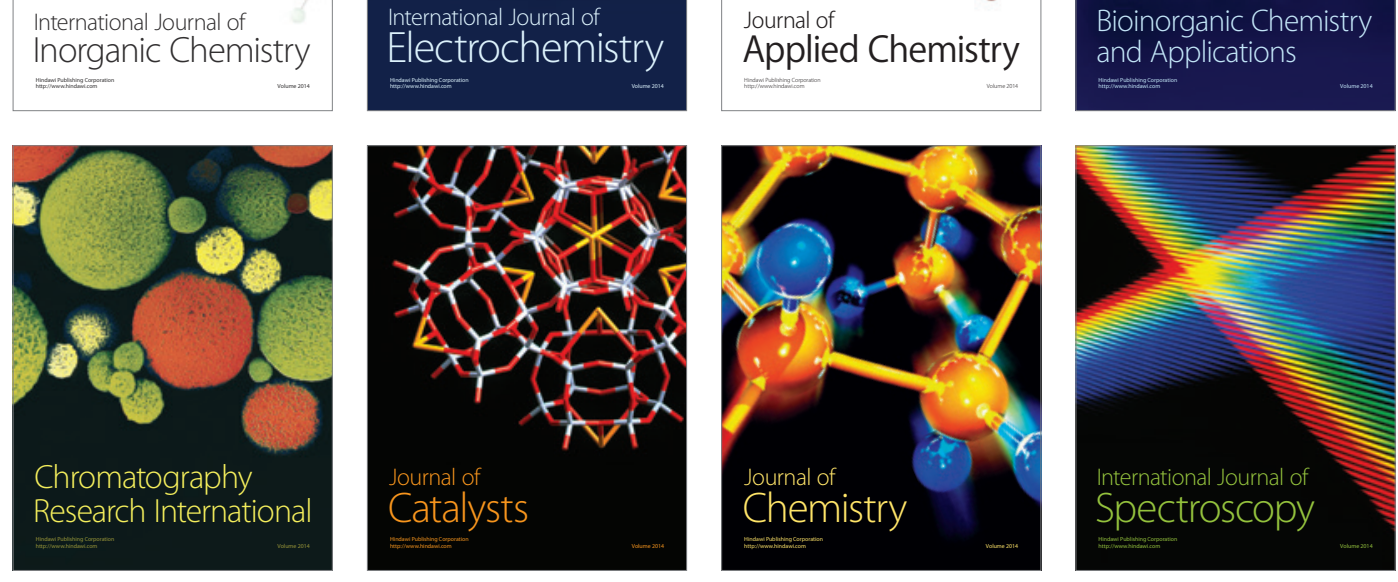